\title{
Ultrafast Population Encoding by Cortical Neurons
}

\author{
Tatjana Tchumatchenko, ${ }^{1,2}$ Aleksey Malyshev, ${ }^{3,4}$ Fred Wolf, ${ }^{1}$ and Maxim Volgushev ${ }^{3,4,5}$ \\ ${ }^{1}$ Max Planck Institute for Dynamics and Self-Organization and Bernstein Center for Computational Neuroscience, D-37073 Goettingen, Germany, \\ ${ }^{2}$ Collaborative Research Center 889 Cellular Mechanisms of Sensory Processing, D-37075 Goettingen, Germany, ${ }^{3}$ Institute of Higher Nervous Activity and \\ Neurophysiology, Russian Academy of Sciences, Moscow 117485, Russia, ${ }^{4}$ Department of Psychology, University of Connecticut, Storrs, Connecticut 06269, \\ and ${ }^{5}$ Department of Neurophysiology, Ruhr University Bochum, D-44801 Bochum, Germany
}

The processing speed of the brain depends on the ability of neurons to rapidly relay input changes. Previous theoretical and experimental studies of the timescale of population firing rate responses arrived at controversial conclusions, some advocating an ultrafast response scale but others arguing for an inherent disadvantage of mean encoded signals for rapid detection of the stimulus onset. Here we assessed the timescale of population firing rate responses of neocortical neurons in experiments performed in the time domain and the frequency domain in vitro and in vivo. We show that populations of neocortical neurons can alter their firing rate within $1 \mathrm{~ms}$ in response to somatically delivered weak current signals presented on a fluctuating background. Signals with amplitudes of miniature postsynaptic currents can be robustly and rapidly detected in the population firing. We further show that population firing rate of neurons of rat visual cortex in vitro and cat visual cortex in vivo can reliably encode weak signals varying at frequencies up to $\sim 200-300 \mathrm{~Hz}$, or $\sim 50$ times faster than the firing rate of individual neurons. These results provide coherent evidence for the ultrafast, millisecond timescale of cortical population responses. Notably, fast responses to weak stimuli are limited to the mean encoding. Rapid detection of current variance changes requires extraordinarily large signal amplitudes. Our study presents conclusive evidence showing that cortical neurons are capable of rapidly relaying subtle mean current signals. This provides a vital mechanism for the propagation of rate-coded information within and across brain areas.

\section{Introduction}

Within 150-200 ms, humans can process complex natural images and relate them to the visual world (Thorpe et al., 1996). In a color-discrimination task, monkeys can make perceptual decisions even within $30 \mathrm{~ms}$ (Stanford et al., 2010). To perform cognitive tasks requiring interactions between multiple brain regions in such short time intervals, neuronal ensembles must be able to rapidly detect and transmit input changes. Sensory stimuli can reach the cortex quickly, e.g., within 5-10 ms in the somatosensory system (Swadlow and Hicks, 1996). However, the mechanisms governing the speed of intracortical communication are poorly understood and widely debated (Silberberg et al., 2004; Koendgen et al., 2008; London et al., 2010). Recently, London et al. (2010) found that cortical neurons are extremely sensitive to changes of their input: injection of a subtle $25 \mathrm{pA}$ current into a single cortical neuron can change population firing rate in a local

\footnotetext{
Received May 2, 2011; revised June 14, 2011; accepted June 28, 2011

Author contributions: T.T., A.M., F.W., and M.V. designed research; T.T., A.M., and M.V. performed research; T.T., A.M., and M.V. analyzed data; T.T., A.M., F.W., and M.V. wrote the paper.

This work is supported by Bundesministerium fuer Bildung und Forschung Grants 01GQ0430, 01GQ1005B, 01GQ07113, and 01GQ07112 (F.W., M.V.), German-Israeli Foundation Grant 906-17.1/2006 (F.W., M.V.), University of Connecticut startup funds (M.V.), Federal Program of Russian Department of Education and Russian Foundation for Basic Research (A.M.), Goettingen Graduate School for Neurosciences and Molecular Biosciences (T.T.), and the Max Planck Society (T.T., F.W.). We are grateful to M. Chistiakova, J. Chrobak, A. Frolov, I. Fleidervich, M. Gutnick, H. Read, and H. Swadlow for fruitful discussions.

The authors declare no competing financial interests.

Correspondence should be addressed to Maxim Volgushev, Department of Psychology, University of Connecticut, 406 Babbidge Road, Unit 1020, Storrs, CT 06269-1020. E-mail: maxim.volgushev@uconn.edu.

DOI:10.1523/JNEUROSCI.2182-11.2011

Copyright $\odot 2011$ the authors $\quad 0270-6474 / 11 / 3112171-09 \$ 15.00 / 0$
}

cortical circuit, but the cellular basis of this remarkable sensitivity and the response timescale is unknown. Theoretically, it is understood that an input signal can be communicated to a neuronal population via two channels. First, a current can be added to the input of all neurons in a population, thus leading to the change of the mean input current. This strategy is plausible for neuronal communication, because a change of the mean current in postsynaptic neurons is the primary effect of synaptic transmission. Second, the variance of input current fluctuations can be changed, such that the signal modulates the variance of the input fluctuations in all neurons, similar to the amplitude modulation strategy widely used in radio communication. Indeed, in the neocortex, the changes in the activity of excitatory and inhibitory populations of neurons can accurately track each other (Okun and Lampl, 2008), such that excitation and inhibition remain balanced. In this case, a perturbation to the network would result only in a change of input variance to each neuron but would change little the mean input current. Thus, changing the variance of the input may represent an additional way of communication between neuronal populations (Lindner and Schimansky-Geier, 2001; Silberberg et al., 2004). Which of the two signal encoding strategies may underlie the rapid communication between populations of cortical neurons? Theoretical analysis suggests that changes of the input mean can mediate fast population responses of the leaky integrate and fire (LIF) model neurons and other models with rapid action potential initiation (Fourcaud-Trocmé et al., 2003). At the same time, an early study suggested that the variance encoding strategy permits extremely fast population rate encoding of strong alternations of the variance of the input to 
neocortical neurons (Silberberg et al., 2004). Here, we show that (1) populations of visual cortex neurons respond immediately to subtle $20 \mathrm{pA}$ change of mean input current in the soma, (2) populations of cortical neurons in vivo can encode fast varying signals up to $200-300 \mathrm{~Hz}$ in their firing, and (3) populations consisting of a few thousand neurons can reliably detect small changes of mean input current within the first few milliseconds after stimulus onset.

\section{Materials and Methods}

All experimental procedures used in this study were in accordance with the guidelines published in the European Communities Council Directive (86/609/EEC, 1986) and conformed to National Institutes of Health regulations. Experimental protocols were approved by the respective local animal welfare committees (Bezirksregierung Arnsberg, Germany, and Institutional Animal Care and Use Committee of University of Connecticut).

In vitro intracellular recordings were made in slices of rat visual cortex. The details of slice preparation and recording were similar to those previously used (Volgushev et al., 2000). The Wistar rats (P21-P28; Harlan) of either sex were anesthetized with isoflurane (Baxter) and decapitated, and the brain was rapidly removed. One hemisphere was mounted onto an agar block, and 350- $\mu \mathrm{m}$-thick sagittal slices containing the visual cortex were cut with a vibratome (Leica) in ice-cooled oxygenated solution. After cutting, the slices were placed into an incubator where they recovered for at least $1 \mathrm{~h}$ at room temperature before transferring them to the recording chamber. The solution used during the preparation of the slices had the same ionic composition as the perfusion/extracellular solution. It contained (in mM) $125 \mathrm{NaCl}, 2.5 \mathrm{KCl}, 2 \mathrm{CaCl}_{2}, 1 \mathrm{MgCl}_{2}, 1.25$ $\mathrm{NaH}_{2} \mathrm{PO}_{4}, 25 \mathrm{NaHCO}_{3}$, and $25 \mathrm{D}$-glucose and was bubbled with $95 \% \mathrm{O}_{2}$ and $5 \% \mathrm{CO}_{2}$. Recordings were made with the slices in submerged conditions at $28-32^{\circ} \mathrm{C}$. Temperature in the recording chamber was monitored with a thermocouple positioned close to the slice, $2-3 \mathrm{~mm}$ from the recording site. Whole-cell recordings using patch electrodes were made from layer $2 / 3$ pyramidal neurons, selected under visual control using Nomarski optics and infrared video microscopy. The patch electrodes were filled with K-gluconate-based solution (in mM: $130 \mathrm{~K}$-gluconate, 20 $\mathrm{KCl}, 4 \mathrm{Mg}$-ATP, $0.3 \mathrm{Na}_{2}$-GTP, $10 \mathrm{Na}$-phosphocreatine, and 10 HEPES) and had a resistance of $4-6 \mathrm{M} \Omega$. All recordings were performed using the bridge mode of Axoclamp-2A amplifier (Molecular Devices). After amplification and low-pass filtering at $10 \mathrm{kHz}$, data were digitized at $20 \mathrm{kHz}$ and fed into a computer (Pentium4; Digidata 1440A interface and pClamp software; Molecular Devices). The recorded membrane potential responses to injected current were processed offline in Matlab (MathWorks). For each cell and each frequency, spikes were detected in membrane potential traces as positive zero crossings, and their times extracted as $\left\{t_{j}\right\}, j=1 \ldots N$. Current injections lasted $46 \mathrm{~s}$ and were separated by a recovery period of $60-100 \mathrm{~s}$. In some experiments, synaptic transmission was blocked by adding $25 \mu \mathrm{M}$ APV, $5 \mu \mathrm{M}$ DNQX, and 80 $\mu \mathrm{M}$ PTX to the extracellular solution. All chemicals were obtained from Sigma, unless stated otherwise.

In vivo intracellular recordings were made in adult cats of either sex $(3.0-4.5 \mathrm{~kg})$. Surgery and animal maintenance were similar to those used in our previous studies (Volgushev et al., 2002). Anesthesia was induced with a mixture of ketamine hydrochloride (Ketanest, $0.3 \mathrm{ml} / \mathrm{kg}$, i.m.; Parke-Davis) and Rompun ( 0.08 ml/kg, i.m.; Bayer). Surgery was started after stable anesthesia with complete analgesia was achieved. Sometimes this required additional doses of the anesthetic. After tracheal and arterial cannulations, the animal was placed in a stereotaxic frame, the skull was exposed, and a craniotomy ( $\sim 5 \mathrm{~mm}$ diameter) was done over area 17 of the visual cortex centered at P4/L3 (Horsley-Clark). A brass cylinder (20 $\mathrm{mm}$ diameter) was cemented over the opening. The holder for hydraulically driven micromanipulator (Narishige Instruments) was mounted onto the skull with screws and dental cement. All wound edges and pressure points were treated with a local anesthetic (Xylocaine; Astra) every 5-8 h. Muscle relaxation with alcuronium chloride (Alloferin; ICN Pharmaceuticals) and artificial respiration were started either at this point or earlier during the surgery to avoid respiratory depression attrib- utable to additional doses of the anesthetic. Thereafter, adequate anesthesia was maintained throughout the experiment by a gas mixture of $\mathrm{N}_{2} \mathrm{O} / \mathrm{O}_{2}$ (70:30) and 0.2-0.4\% halothane (Eurim-Pharm). Artificial respiration was performed with a cat/rabbit ventilator (model 6025; Ugo Basile, Biological Research Apparatus). The volume $\left(20-40 \mathrm{~cm}^{3}\right)$ and the rate of stroke (7-15 per minute) were adjusted to maintain end-tidal $\mathrm{CO}_{2}$ between 3.5 and $4.0 \%$. End-tidal $\mathrm{CO}_{2}$, body temperature, heart rate, blood pressure, and EEG were continuously monitored. Body temperature was maintained $\sim 37-38^{\circ} \mathrm{C}$. Fluid replacement was achieved by the intra-arterial administration of $6 \mathrm{ml}$ of Ringer's solution containing $1.25 \%$ glucose per hour. Paralysis was maintained by intra-arterial infusion of alcuronium chloride $\left(0.15 \mathrm{mg} \cdot \mathrm{kg}^{-1} \cdot \mathrm{h}^{-1}\right)$ in Ringer's solution. The experiments lasted usually $2-4 \mathrm{~d}$. At the end of the experiment, animals were killed with an overdose of anesthetics. In vivo intracellular recordings from visual cortical neurons were made with sharp electrodes filled with $2 \mathrm{M}$ potassium acetate. Electrode resistance was 80-120 M $\Omega$. Recordings were made using the bridge mode of Axoclamp-2B amplifier (Molecular Devices). After amplification and low-pass filtering at 10 $\mathrm{kHz}$, the data were digitized at $20 \mathrm{kHz}$ and stored on a computer (Pentium4; Digidata 1322A; Molecular Devices). Current injections lasted $30 \mathrm{~s}$ and were separated by a recovery period of $60-100 \mathrm{~s}$. For each cell and each frequency, spikes were detected in membrane potential traces with the same methods as in the in vitro recordings.

Assessing the frequency response function of neuronal populations. To assess the frequency response of neuronal populations, we have somatically injected currents that were composed of a sinusoid signal of frequency $f$ immersed in different realizations of a noise for in vitro experiments or without added noise in vivo. A constant direct current was added to maintain a target firing rate of $\sim 5 \mathrm{~Hz}$. Currents were injected in 30-46 s episodes, with 60-100 s intervals between the injections. For each recording condition, in vitro with correlation time constant of injected noise $\tau_{I}=5 \mathrm{~ms}$ or $\tau_{I}=50 \mathrm{~ms}$ or in vivo, currents were injected in $n=4 \ldots 10$ different neurons. The vector strength $r$ characterizes phase locking of firing of the neurons to the periodic stimulus. $r$ was computed using all recordings for a frequency $f$ in each recorded cell individually and subsequently averaged across cells. In each cell, responses to several frequencies were recorded. The number and length of recordings were as follows. In vitro recordings with $\tau_{I}=5 \mathrm{~ms}$ were obtained in nine cells, in which each cell contributed totally $\sim 9000$ spikes to frequencies $f(f=3,5,50,110,230,370$, and $515 \mathrm{~Hz})$. In vitro recordings with $\tau_{I}=50 \mathrm{~ms}$ were obtained in 10 cells, in which each cell contributed $\sim 9000$ spikes to frequencies $f(f=3,5,50,110,230,370$, and $515 \mathrm{~Hz}$ ). In vivo recordings were obtained in four cells, in which each cell contributed $\sim 1000$ spikes to individual frequencies $f(f=3,13,50,110$, $200,300,400,500$, and 600$)$. Parameters of injected currents were adjusted to obtain similar amplitudes of membrane potential fluctuations and similar firing rates $(v)$ in all in vivo and in vitro experiments: in vitro $\tau_{I}=5 \mathrm{~ms}$, membrane potentials fluctuations, $-56 \pm 6.2 \mathrm{mV}, v=4.5 \pm$ $1.2 \mathrm{~Hz}$; in vitro $\tau_{I}=50 \mathrm{~ms}$, membrane potentials fluctuations, $-48.1 \pm$ $7.4 \mathrm{mV}, v=5.2 \pm 0.9 \mathrm{~Hz}$; in vivo membrane potentials fluctuations, $-49.1 \pm 7.1 \mathrm{mV}, v=5.6 \pm 2.2 \mathrm{~Hz}$.

To determine the statistical significance of phase locking of recorded spikes to periodic input current stimulation, we used a randomization test. This test was performed for spikes recorded for each input frequency $f$ and each cell separately. The value of the experimentally obtained vector strength $r$ was compared with values obtained for datasets of the same size but randomized phase. Ten thousand independent realizations of random sets $\left\{x_{j}^{\prime}\right\}$ where each $x_{j}^{\prime} \in N(0,1), j=1 \ldots N$ (number of spikes recorded in a cell in response to the input frequency $f$ ) were used to generate 10,000 realizations of phase randomized sets of spike times $t_{j}^{\prime}=$ $\left(\bmod \left(t_{j} \cdot f, 1\right)+x_{j}^{\prime}\right) / f$. This procedure keeps the number of spikes equal to that obtained experimentally but eliminates any original phase preference. For each of the randomized sets of spike times, the corresponding vector strength was calculated $r_{s}^{\prime}=\operatorname{abs}\left(\sum_{j=1}^{N} \exp \left(i 2 \pi f t_{j}^{\prime}\right)\right) / N$. For the $r_{s}^{\prime}$ distribution, we calculated the 95th percentile, which is the value below which $95 \%$ of the randomly drawn $r_{s}^{\prime}$ can be found. The probability to obtain by chance a value above the 95 th percentile is $5 \%$. The 95 th percentile of the $r_{s}^{\prime}$ distribution was taken as the single-cell significance level for the modulation frequency $f$. The maximal 95th percentile value ob- 
A

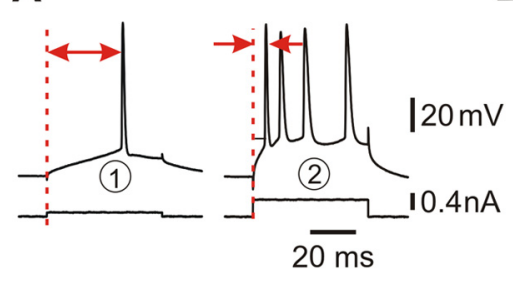

C

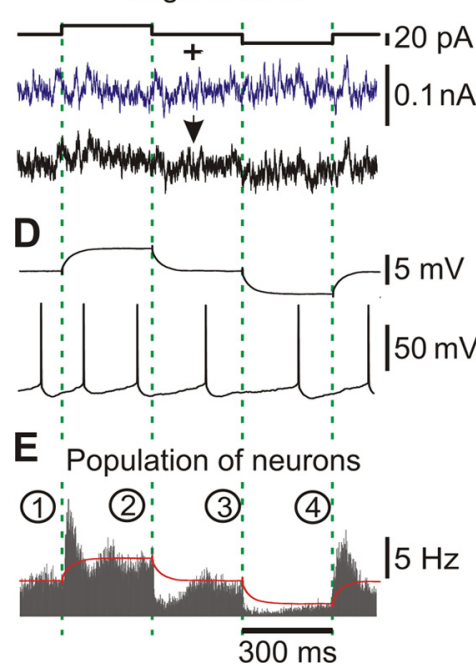

B

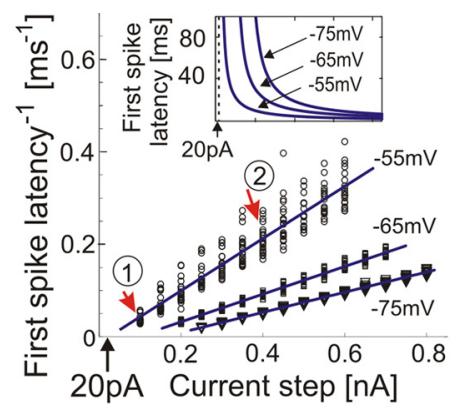

$\mathbf{F}$

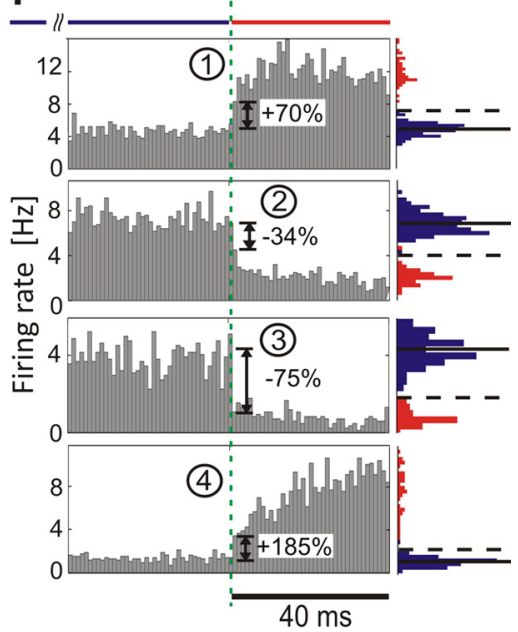

Figure 1. Encoding of current steps by single neurons and populations in vitro. $A$, Responses of a $L 2 / 3$ neuron to 0.1 and $0.4 \mathrm{nA}$ steps. Red arrows, First spike latency. $\boldsymbol{B}$, Inverse first spike latency versus step amplitude $(N=20$ for each step) and linear fits (blue lines). Red arrows, Responses in $\boldsymbol{A}$. Inset, First spike latency versus step amplitude as derived from linear fits of the inverse first spike latency (same current scale). C, Current steps (top) and current steps immersed in fluctuating current ( $\tau_{l}=5 \mathrm{~ms}$ ) (bottom). To ensure weak stimulation, we chose current steps of $20 \mathrm{pA}$, which in $\boldsymbol{A}$ correspond to an approximated latency $>100 \mathrm{~ms}$. $\boldsymbol{D}$, Membrane potential response to subthreshold current steps as in $\boldsymbol{C}$ (top) (average, $N=20$ ) and to current in $\boldsymbol{C}$ (bottom). $\boldsymbol{E}$, Population firing in response to steps immersed in different realizations of fluctuating current, bin size $1 \mathrm{~ms}$. Peristimulus time histogram is constructed over all repetitions pooled from all 15 cells ( $\sim 175 \mathrm{~min} ; \sim 52,000$ spikes) by aligning the evoked spike trains with the onset of current steps. Red line, Membrane potential from $\boldsymbol{D}$ (top). $\boldsymbol{F}$, Zoom-in on responses in $\boldsymbol{E}$. Blue and red histograms denote distributions of spike counts in $1 \mathrm{~ms}$ bins $120 \mathrm{~ms}$ before (blue) and $40 \mathrm{~ms}$ after (red) each step. In the distributions before the step, solid horizontal lines denote the mean and dashed lines 3 SD. Dashed green lines in $\boldsymbol{C}-\boldsymbol{F}$ indicate the onset of steps.

tained among cells recorded with the same modulation paradigm are shown in the respective figure as significance levels.

Effect of step-like current change on voltage statistics. To assess the effect of step current change on voltage statistics, we calculated the voltage distributions in each period of the step protocol (intervals of $1 \mathrm{~s}$ for data in Fig. 3, and intervals of $300 \mathrm{~ms}$ for data in Figs. 1,2). When calculating the distributions, spikes were truncated at $-20 \mathrm{mV}$. In all in vitro experiments, voltage distributions had a mean of -45 to $-55 \mathrm{mV}$ and an SD of 3-7 $\mathrm{mV}$, which is within the previously reported in vivo range (Destexhe et al., 2003; Volgushev et al., 2006). Steps of the mean current of $\pm 20 \mathrm{pA}$ delivered with a noise component $\sigma \eta(t)$ (data from Figs. 1, 3C) or with intrinsic noise only (see Fig. 2) changed the membrane potential mean by $\sim 1-2 \mathrm{mV}$, but its SD remained same or changed by $<0.5$ $\mathrm{mV}$. Changes in the input current variance $(\sigma \rightarrow 1.5 \sigma)$ (see Fig. $3 A$ ) lead to $<0.5 \mathrm{mV}$ changes of mean voltage and $\sim 0.2-0.5 \mathrm{mV}$ changes in SD. Larger changes in the input current variance $(\sigma \rightarrow 3 \sigma)$ (see Fig. $3 B$ ) did not significantly alter the mean but clearly increased the voltage $\mathrm{SD}$ by $\sim 1-2 \mathrm{mV}$.

Step onset detection. To quantify the speed of step onset detection, we assume a theoretical decoder that reports a step change of input current if the population firing rate falls outside the $95 \%$ confidence boundary of pre-step distribution. We calculate the probability of step detection as a function of the number of neurons that receive the common current step (equivalent to the number of realizations $N$ ) and time delay $T$ after the step. To obtain the probability of step detection by $N$ neurons within a time interval $T_{\mathrm{ms}}$ after the step onset, we composed 1000 trial sets; each consisting of $N$ randomly selected sweeps. In each trial set, we determined whether the spike count in the interval $T$ after step onset fell outside the $95 \%$ percentile of the corresponding pre-step distribution. The number of trial sets, which fulfill this condition, provides an estimate of the probability for a population of $N$ neurons to detect the step change within a time $T$ after step onset. The probability to detect a step change within the time $t$ is equivalent to the cumulative probability to encounter a spike count larger than the $95 \%$ percentile (or smaller than the $5 \%$ percentile if the firing rate is decreased) of the spike count distribution before the step onset. For an increase of firing rate, this can be computed as $1-\operatorname{CDF}\left(P\left(N, v_{2} t\right)\right.$, Percentile $\left.\left(P\left(N, v_{1} t\right), 0.95\right)\right)$, where CDF is the cumulative distribution function. For the decrease of firing rate, we compute detection probability as $\operatorname{CDF}\left(P\left(N, v_{2}\right)\right.$, Percentile $\left.\left(P\left(N, v_{1} t\right), 0.05\right)-1\right)$. Here, $P\left(N, v_{1} t\right)$ is the spike count probability distribution, where $P$ is the probability to fire a spike given the average population rate $\nu_{1}, N$ the number of neurons is the initial probability distribution of spike counts, and $P\left(N, v_{2} t\right)$ is the distribution of spike counts after the step change.

\section{Results}

Layer 2/3 pyramidal neurons that mediate computation within and communication between different cortical areas (Gilbert and Wiesel, 1979) are particularly relevant for sensory processing. To directly test how populations of $\mathrm{L} 2 / 3$ cortical neurons respond to subtle input changes, we examined their firing rate dynamics in response to small-amplitude current steps immersed in a fluctuating background. Currents $I(t)$ for in vitro injection into the soma through a patch electrode were digitally synthesized offline using the following equation:

$$
\begin{aligned}
& I(t)=I_{0}+\sigma \eta(t)+I_{m} \cdot \operatorname{Step}(t), \\
& \text { where } \operatorname{Step}(t)= \begin{cases}1 & \text { if } 300 \mathrm{~ms} \leqslant \bmod (t, 1.2 \mathrm{~s})<600 \mathrm{~ms}, \\
-1 & \text { if } 900 \mathrm{~ms} \leqslant \bmod (t, 1.2 \mathrm{~s})<1.2 \mathrm{~s}, \\
0 & \text { else }\end{cases}
\end{aligned}
$$

Here and in all following experiments, $I_{0}$ is a constant current set to maintain a target firing rate of $5 \mathrm{~Hz} . \eta(t)$ is an OrnsteinUhlenbeck process with 0 mean, unit variance, and correlation time $\tau_{I}=5 \mathrm{~ms}$, and $\sigma$ is the SD of the resulting background current noise. This noise component $\sigma \eta(t)$ mimics the effective somatic current produced by a large number of balanced excitatory and inhibitory synaptic inputs in vivo (Destexhe et al., 2003). $I_{\mathrm{m}} \cdot \operatorname{Step}(t)$ describes the signal, in this case positive and negative steps of amplitude $I_{\mathrm{m}}$ and duration of $300 \mathrm{~ms}$ that were interleaved with no stimulus (noise only) periods of $300 \mathrm{~ms}$. One stimulation cycle lasted for $1.2 \mathrm{~s}$, with time course defined by 
$\operatorname{Step}(t)$ in Equation 1 , where $\bmod (t, 1.2 \mathrm{~s})$ denotes $t$ modulo the period length of $1.2 \mathrm{~s}$ (Fig. 1C). To guarantee weak stimulation, the amplitude was set to $I_{\mathrm{m}}=20$ $\mathrm{pA}$, which corresponds to amplitudes of unitary postsynaptic currents resulting from the activation of a single excitatory or inhibitory presynaptic fiber (Stern et al., 1992; Hestrin, 1993), and is more than one order of magnitude weaker than a current step necessary to depolarize a typical neuron from the resting potential to firing threshold (Fig. 1B). When applied at subthreshold potentials, steps of this small amplitude led to an approximated first spike latency $>100 \mathrm{~ms}$, even for holding potentials close to the spike threshold $(-55 \mathrm{mV}$ ) (Fig. 1A,B). A $20 \mathrm{pA}$ up or down step in the current mean evoked a mean membrane potential change of $\sim 1-2 \mathrm{mV}$ and left membrane potential fluctuation variance unaffected. To test whether populations of neocortical neurons can detect these small changes at their inputs, we injected currents with different realizations of the noise component in $15 \mathrm{~L} 2 / 3$ pyramidal cells (Fig. $1 E$ ). Cell firing during individual responses to current injection did not show an obvious relation to the mean current step (Fig. 1D, bottom trace). The population firing rate, however, clearly changed with the onset and offset of step stimuli, as revealed by the peristimulus time histogram of the spike times constructed over all repetitions pooled from all 15 cells. It would be plausible to expect that the low-pass filtering of input currents by the membrane RC (resistor-capacitor) circuit should carry over to the firing rate dynamics. However, in response to the current steps presented on an in vivo-like background, the population firing rate already changed within the first 1-2 ms after stimulus onset, substantially faster than the mean membrane potential of the neuron (Fig. $1 E$ ). Intuitively, ultrafast population response can be understood using the following oversimplified reasoning. In a population of firing neurons subject to random input, there are always some neurons that are just about to fire and any additional depolarization will immediately bring them over the threshold, thus producing an instantaneous population response. In these "early responders," an action potential is triggered by the initial subtle depolarization at the step onset, before the whole neuron is charged and membrane potential reaches the steady state. Rapid change of the population firing rate at the onset and offset of current steps is clearly seen at zoom-in of the initial portions of step responses (Fig. $1 F$ ). The population rate rapidly conveyed both positive and negative changes of the mean current and could increase by $185 \%$ or decrease by $75 \%$ within $1 \mathrm{~ms}$ (Fig. $1 \mathrm{~F}$ ). How important is the presence of a substantial, in vivo-like background activity for the ultrafast changes of population firing rate in response to subtle changes of the mean input current? To clarify the influence of the noise component in fast population responses, we conducted control experiments with no noise component added $[\eta(t)=0$ ] (Fig. $2 A, B$ ). In this case, only low intrinsic noise of in vitro preparation, e.g., attributable to spontaneous transmitter release and channel noise, was present (Steinmetz et al., 2000; Jacobson et al., 2005). Background firing was kept at $\sim 5 \mathrm{~Hz}$ by injecting a constant depolarizing current.
Control, without additional noise $(\eta(t)=0)$
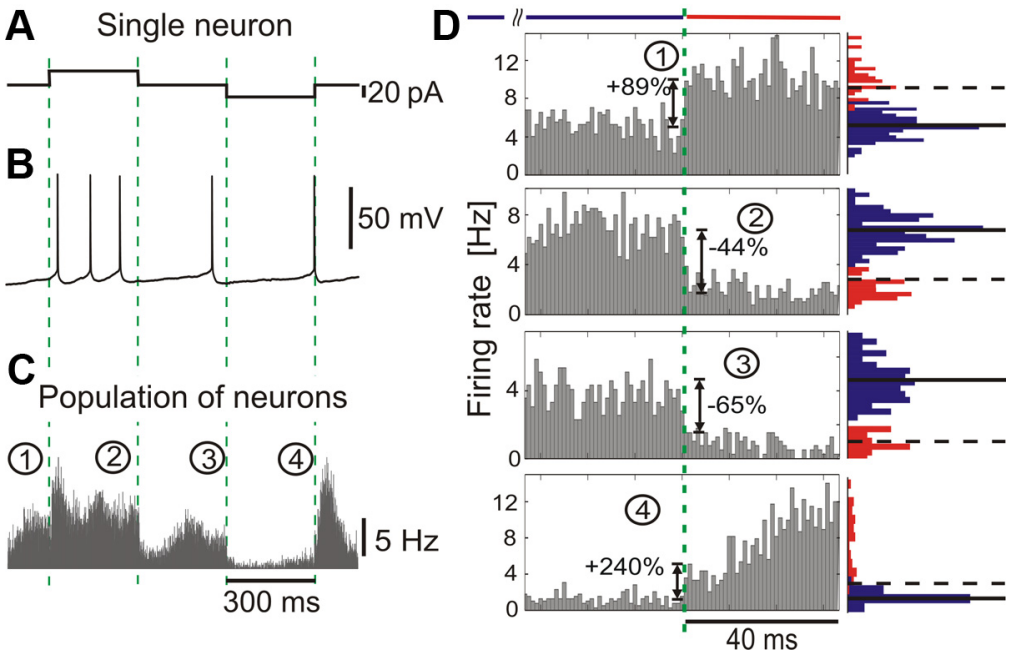

$40 \mathrm{~ms}$

Figure 2. Encoding of current steps by single neurons and populations in vitro under minimal noise conditions. $\boldsymbol{A}$, Current steps. $\boldsymbol{B}$, An example of membrane potential response to current in $\boldsymbol{A}$. $\boldsymbol{C}$, Population response to current steps with intrinsic noise only and blue horizontal bars. In the distributions before the step, horizontal lines show the mean rate (solid) and 3SD (dashed). Dashed green lines in $\boldsymbol{A}-\boldsymbol{D}$ indicate the step onset, same notation as in Figure 1.

Positive and negative current steps evoked rapid, abrupt changes of population firing rate with a magnitude similar to that observed in experiments with added noise (compare Figs. $1 E, F$ and $2 C, D)$. Thus, fast responses of neuronal populations to current steps were robust phenomena, observed both in the presence of in vivo-like membrane potential fluctuations, as well as with minimal, intrinsic-only, noise. Results presented in Figures 1 and 2 clearly demonstrate that populations of layer $2 / 3$ neocortical neurons can respond to step-like changes of the mean input very rapidly, on a millisecond timescale. This is the first demonstration of fast detection of weak mean encoded stimuli in the time domain. Notably, previous studies of the speed of cortical population encoding performed in the time domain and in the frequency domain reported controversial results. The only study conducted so far in the time domain in layer 5 pyramids used current steps immersed in an almost-white Gaussian noise background with $\tau_{I}=0.25 \mathrm{~ms}$ or in noise background with an unspecified time constant that was recorded during elevated synaptic activity induced by application of potassium channel blocker 4-AP (Silberberg el al. 2004). This study reported a slow response timescale for changes of the current mean and concluded that encoding of mean changes is intrinsically slow and only variance-encoded signals can be detected fast. However, this result could be biased because of predominant use of physiologically unrealistic almost-white noise that has been associated in theoretical studies with a slow response timescale on the order of the membrane time constant (Brunel et al., 2001; Lindner and Schimansky-Geier, 2001; Ostojic et al., 2009). Interestingly, frequency domain studies reported that layer 5 pyramidal neurons can encode remarkably fast stimuli up to $200-300 \mathrm{~Hz}$ in the presence of background noise correlated either on a short $\left(\tau_{I}=5\right.$ $\mathrm{ms}$ ) or a long timescale (Koendgen et al., 2008; Higgs and Spain, 2009). This suggests that mean signals can be detected at a limiting timescale of $\sim 1 /(2 \pi 200 \mathrm{~Hz})<1 \mathrm{~ms}$. To resolve this contradiction, it is necessary to study encoding of mean and variancecoded signals under the same experimental conditions and in 


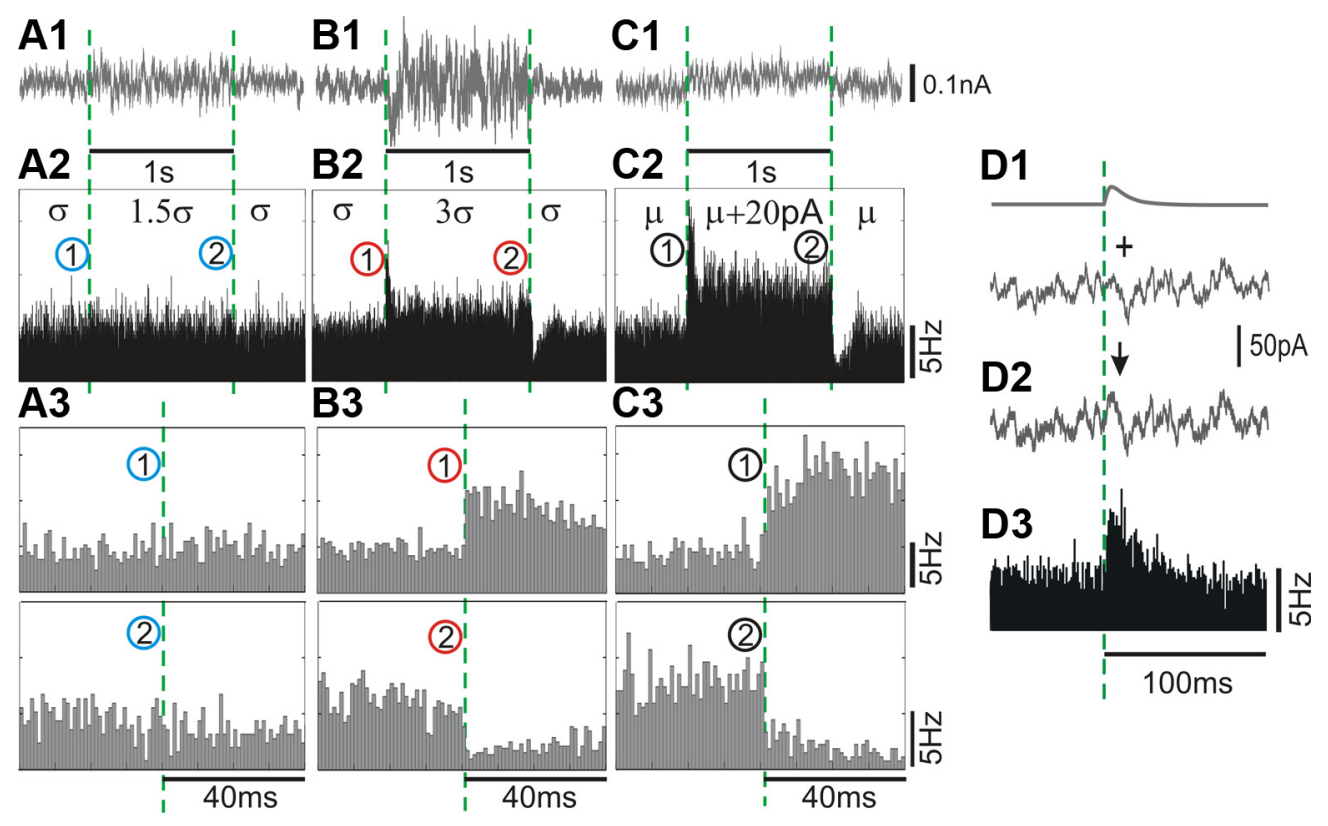

Figure 3. Population response to changes in variance $(\boldsymbol{A}, \boldsymbol{B})$ or mean $(\boldsymbol{C})$ of the input current. $\boldsymbol{A}-\boldsymbol{C}$, Examples of fluctuating currents $\left(\tau_{l}=5 \mathrm{~ms}\right)$ with step-like changes in the SD $\sigma$ by $50 \%$ ( $\boldsymbol{A} 1$, $\sigma \rightarrow 1.5 \sigma \rightarrow \sigma)$, by $200 \%(B 1, \sigma \rightarrow 3 \sigma \rightarrow \sigma)$ or step-like changes of the mean current $\mu(\mathbf{C} 1, \mu \rightarrow \mu+20 \mathrm{pA} \rightarrow \mu)$. Population firing rate changes in response to injection of currents as in A1-C1, 2530 realizations (A2), 5214 realizations (B2), and 2706 realizations (C2). A3-C3, Zoom-in of responses to the onset and offset of step-changes in (A2-C2). D, EPSC-like current pulse immersed in fluctuating background $(\mathbf{D 1}, \mathbf{D 2})$ is reliably detected by a population of neurons $(\mathbf{D 3}, 7600$ realizations). In all subfigures, dashed green lines indicate the onset of steps or EPSCs; bin size, $1 \mathrm{~ms}$.

neurons of the same type. Therefore, we next studied responses of layer $2 / 3$ pyramidal neurons to variance-coded signals in time domain and to mean-coded signals in the frequency domain.

\section{Low susceptibility to variance coded signals}

Because the fluctuations in the activity of excitatory and inhibitory populations accurately track each other in cortical networks (Okun and Lampl, 2008; Renart et al., 2010; Ecker et al., 2010), a perturbation to the network can result predominantly in a change of input current variance, with little or no change in its mean. Theoretical studies showed that changing the input current variance may represent a viable way of communication between neuronal populations (Lindner and Schimansky-Geier, 2001; Silberberg et al., 2004; Fourcaud-Trocmé and Brunel, 2005; Naundorf et al., 2005b). To test this conjecture and to directly compare the two encoding strategies in L2/3 pyramidal neurons, we studied the firing rate dynamics in vitro in response to steplike changes in the variance of the fluctuating input current:

$$
\begin{aligned}
& I(t)=I_{0}+\sigma \eta(t) \cdot\left[1+\varepsilon_{v} \cdot \operatorname{Box}(t)\right] \\
& \text { where } \operatorname{Box}(t)= \begin{cases}1 & \text { if } 0 \leqslant \bmod (t, 2 \mathrm{~s})<1 \mathrm{~s}, \\
0 & \text { else }\end{cases}
\end{aligned}
$$

As in the experiments described above, the constant current component $I_{0}$ was used to achieve a target firing rate of $5 \mathrm{~Hz}$ and $\eta(t)$ was the Ornstein-Uhlenbeck process with $\tau_{I}=5 \mathrm{~ms}$. $\varepsilon_{v}$ is the magnitude of the SD increase, $\varepsilon_{v}=0.5$ or $\varepsilon_{v}=2$ in our experiments. $\bmod (t, 2 \mathrm{~s})$ denotes $t$ modulo the period length of $2 \mathrm{~s}$, so that every second the SD of the fluctuating input was switched between $\sigma$ and $\sigma\left(1+\varepsilon_{v}\right)$. To facilitate a comparison between the variance versus the mean encoding strategies, a protocol with the same time course was repeated for steps of mean current synthesized as follows:

$$
I(t)=I_{0}+\sigma \eta(t)+I_{\mathrm{m}} \cdot \operatorname{Box}(t),
$$

with $I_{\mathrm{m}}=20$ pA. A $20 \mathrm{pA}$ mean current step evoked a mean membrane potential change of $\sim 1-2 \mathrm{mV}$ and left membrane potential fluctuation variance unaffected. The $\varepsilon_{v}=2$ step increased the SD of the membrane potential fluctuations by $\sim 1-2$ $\mathrm{mV}$ but left the mean voltage unaffected. Confirming the results described above, small-amplitude 20 pA steps of the mean current induced pronounced changes of the population firing rate of layer 2/3 neurons, with clear instantaneous firing rate changes at the onset of positive and negative steps (Fig. 3C). In contrast, a step-like increase of the SD of the input current with comparable magnitude $\left(\varepsilon_{v}=0.5\right)$ failed to elicit a measurable firing rate response (Fig. $3 A$ ). When we increased the magnitude of the variance step to $\varepsilon_{v}=2$, which corresponds to a threefold $\sigma$ increase (Fig. $3 B$ ), the increased input current fluctuations elicited a substantial firing rate response. These results are consistent with the large variance changes that were necessary to elicit a firing rate response in neocortical neurons studied in time domain (Silberberg et al., 2004) and in frequency domain (Boucsein et al., 2009), as well as with the firing rate dependence on current variance of cortical neurons (Rauch et al., 2003). In responses to large variance changes, the population firing rate exhibited virtually instantaneous components arising within 1-2 ms after the stimulus onset (Fig. 3B). The instantaneous components of the response to $3 \sigma$ steps were comparable in magnitude and speed with the instantaneous components in responses to small-amplitude steps of the mean current (Fig. $3 B, C$ ). The tonic firing rate during the $3 \sigma$ step changed significantly less than during response to subtle 20 pA mean current steps. These results reveal a substantial difference in firing rate responses of layer $2 / 3$ pyramidal neurons to signals encoded via these two strategies. Minor changes of the mean input current induce a virtually instantaneous change of firing rate at the step onset, followed by a robust change of the stationary firing rate. Thus, both the onset and duration of small changes in the mean input current are reflected in the population 
firing with high temporal precision. In contrast, large changes in the input variance appear to be necessary to affect the population firing rate, whereby the response is predominantly transient with a weak stationary component. These results confirm the previously reported observation that large variance changes are necessary to elicit a population firing rate response (Rauch et al., 2003, their Fig. 4; Silberberg et al., 2004, their Figs. 1B, 4B). However, our results (Fig. 3) also show that, to achieve a same size tonic rate component, much larger changes of the variance than of the mean are needed. Therefore, if during experiments the magnitudes of current variance change and current mean change are adjusted to produce the same increase of the tonic firing rate, the nonlinear responses to very strong variance changes will be compared with responses to small or moderate changes of the mean input current. This bias of the stimuli strength in favor of variance changes could be one of the reasons why previous studies overlooked the fast onset dynamics of responses to mean current changes.

\section{Detection of PSCs by populations of neurons}

To further corroborate fast transmission of subtle mean current signals in cortical neuronal populations, we studied the firing rate dynamics of L2/3 pyramidal neurons in response to EPSC-like currents immersed in a fluctuating background. EPSC-like currents with rise time $1 \mathrm{~ms}$ and decay time $10 \mathrm{~ms}$ were synthesized as $f(t)=\varepsilon_{e}(\exp (-t / 10 \mathrm{~ms})-\exp (-t / 1 \mathrm{~ms}))$, with $\varepsilon_{e}$ chosen such that the peak amplitude of each current pulse was $20 \mathrm{pA}$ (Fig. $3 D 1$ ), and added to a fluctuating background current $\sigma \eta(t)$ every $300 \mathrm{~ms}\left(t_{i}=300 \mathrm{~ms} \cdot i\right.$ with $i \epsilon N ; I_{0}$ and $\sigma \eta(t)$ as in Eqs. 1-3). Injected currents $I(t)$ were synthesized as

$$
I(t)=I_{0}+\sigma \eta(t)+\sum_{i} f\left(t-t_{i}\right) .
$$

The firing rate of a population of cortical neurons changes quickly and robustly in response to the fast, small-amplitude EPSC (Fig. 3D). So far, ability to trigger an immediate spiking response in populations of postsynaptic cells has been demonstrated only for the exceptionally strong cortical synapses with the postsynaptic current amplitudes of $\sim 200 \mathrm{pA}$ or above (Galarreta and Hestrin, 2001). However, the overwhelming majority of cortical synapses are much weaker, with postsynaptic current amplitudes of $\sim 20 \mathrm{pA}$ or smaller (Stern et al., 1992) and PSP amplitudes well below $1 \mathrm{mV}$ (Matsumura et al., 1996, their Tables $2,3)$. Our results show that activity at such weak synapses can be rapidly detected by postsynaptic population of a few thousand neurons on the background of substantial, in vivo-like fluctuations. Thus, a population of neurons receiving fast, smallamplitude EPSC from just one common presynaptic cell can reliably detect a single presynaptic spike and propagate this information to downstream cells. This indicates that the population firing rate response to a single additional spike or a minor current injection, as observed in local cortical circuits (London et al., 2010), can be mediated via the mean-current signaling channel alone.

\section{In vivo and in vitro response to periodic stimuli}

To directly compare the timescale of population responses to step stimuli in the time and in the frequency domain, we measured the frequency response function of layer $2 / 3$ pyramidal neurons. To facilitate a comparison with recent in vitro work in other types of cortical cells (Koendgen et al., 2008; Boucsein et al., 2009), we obtained the frequency response function by measuring the response to each input frequency individually. The resulting frequency response function allows us to identify the bandwidth of reliably encoded frequencies, which is closely connected to the timescale of the rising phase in the population response (Brunel et al., 2001; Fourcaud-Trocmé et al., 2003). Currents $I(t)$ for somatic injection into neurons were composed of a sinusoid signal of frequency $f$ immersed in a noise. A constant current $I_{0}$ was added to maintain target firing rate of $5 \mathrm{~Hz}$ :

$$
I(t)=I_{0}+\varepsilon_{\mathrm{m}} \sin (2 \pi f t)+\sigma \eta(t) .
$$

The noise mimicking in vivo synaptic bombardment was generated as an Ornstein-Uhlenbeck process $\eta(t)$ with a correlation time $\tau_{I}=5 \mathrm{~ms}$ or $\tau_{I}=50 \mathrm{~ms}$, SD $\sigma$ and signal-to-noise ratio $\varepsilon_{\mathrm{m}} /\left(\varepsilon_{\mathrm{m}}+\sigma\right)=0.26$ (Fig. $4 A, B$ ). The ability of neurons to encode signals of frequency $f$ was quantified using the vector strength (Goldberg and Brown, 1969; Joris et al., 2004; Zheng and Escabi, 2008) $r=\operatorname{abs}\left(\sum_{j=1}^{N} \exp \left(i 2 \pi f t_{j}\right)\right) / N$, where $t_{j}$ are the spike times and $N$ the number of spikes. Here, each spike is represented by a vector of unit length and a phase between 0 and $2 \pi$ defined by the spike time modulo the stimulus period. If all spikes are emitted at the same phase of the oscillation cycle, then $r$ is maximal $(r=1)$, indicating a perfect encoding of the input frequency. If spikes occur at random phases, the vector strength is close to zero, indicating that the signal frequency is not encoded in the firing rate. We have assessed the frequency response function of layer $2 / 3$ pyramidal neurons by calculating vector strength in responses to different frequencies $(3-515 \mathrm{~Hz}$ ) (for more details, see Materials 
A
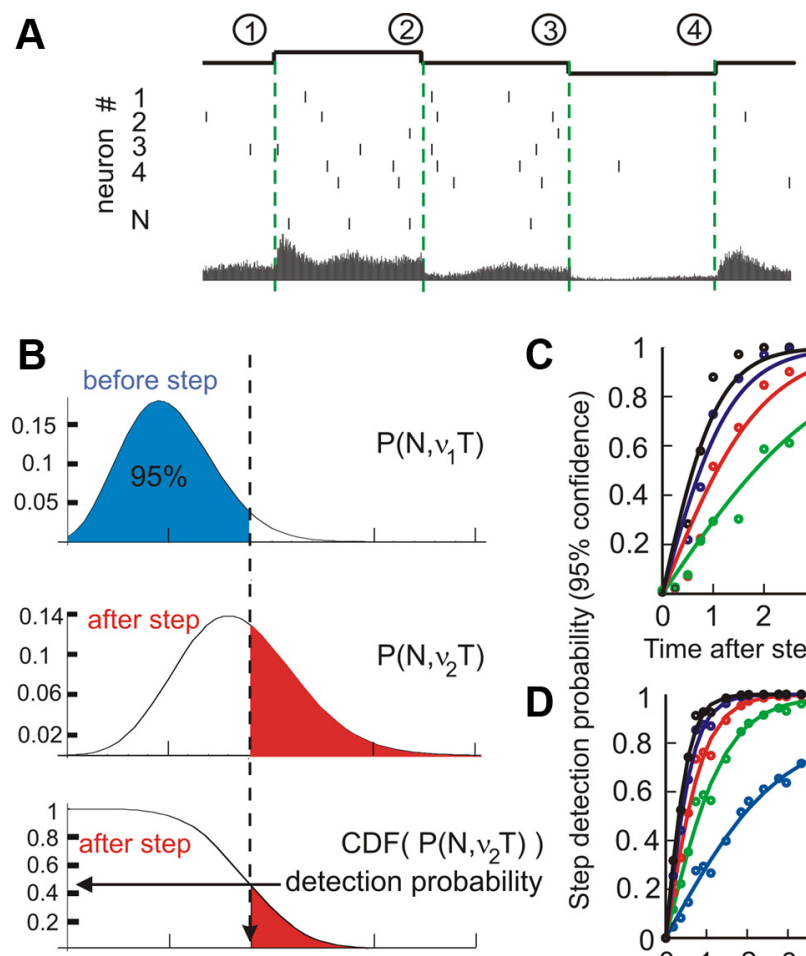

spike count
C

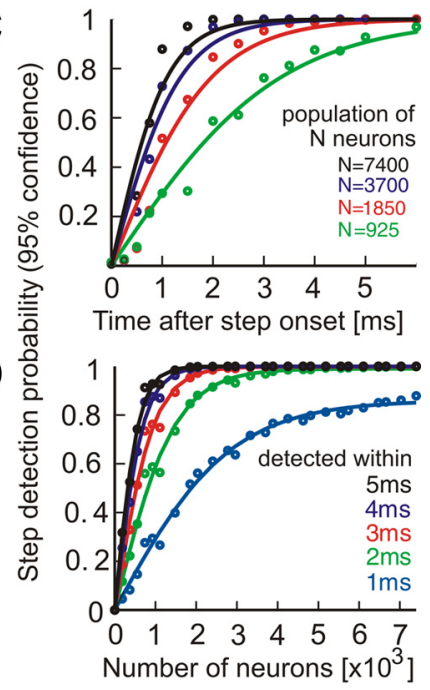

Figure 5. Detection of population firing rate changes induced by changes of the mean input current. $A$, lllustration of step encoding in the population firing rate of $N$ neurons. Top to bottom, Timing of current steps; spike responses of neurons $1 \ldots N$, with vertical bars representing individual spikes and the resulting peristimulus time histogram of the population firing rate (bottom, data from Fig. 1E). $\boldsymbol{B}$, Illustration of a theoretical decoder. Binomial distributions $P\left(N, v_{1} T\right)$ of spike counts before the step, $P\left(N, v_{2} T\right)$ after the step and its cumulative distribution $\operatorname{CDF}\left(P\left(N, v_{2} T\right)\right)$. From the latter, the probability that after-step spike count is outside the $95 \%$ boundary of the pre-step distribution is determined (horizontal arrow). This corresponds to the probability of step detection with $95 \%$ confidence. $C, \boldsymbol{D}$, Probability of step detection versus time after step onset $(\boldsymbol{C})$ and number of neurons $(\boldsymbol{D})$ as calculated from data in Figure $1(-F$ and averaged across conditions (1-4). Circles denote the data points and solid lines denote fits of the form $f(T)=\tanh (b T)$ or $f(M)=a \tanh (b N)$, respectively. Note that a population of 7400 neurons (equivalent to the number of realizations) can detect $88 \%$ of steps within the first $1 \mathrm{~ms}$.

and Methods). Frequency response functions in Figure $4, D$ and $E$, show that that layer $2 / 3$ pyramidal neurons in vitro can reliably encode weak periodic signals up to cutoff frequencies $f_{c} \sim 200-$ $300 \mathrm{~Hz}$ in their population firing rate. Furthermore, input signals varying at frequencies of hundreds of hertz were encoded for both short $\left(\tau_{I}=5 \mathrm{~ms}\right)$ or long $\left(\tau_{I}=50 \mathrm{~ms}\right)$ correlation time constants (Fig. 4D,E, blue and black curves). Can cortical neurons in vivo also encode such high-frequency signals in their population firing? To assess this question, we made intracellular recordings from regular spiking neurons in area 17 of cat visual cortex in vivo and studied their firing rate dynamics in response to injection of sinusoidally modulated currents of amplitude $\varepsilon_{\mathrm{m}}$ $=50 \mathrm{pA}$ and different frequency $f: I(t)=I_{0}+\varepsilon_{\mathrm{m}} \sin (2 \pi f t)$. No noise was added to the injected current because membrane potential fluctuations were produced by synaptic bombardment attributable to the background inactivity in vivo (Fig. 4C). Frequency response function of visual cortex neurons in vivo showed high cutoff frequency of $200-300 \mathrm{~Hz}$ (Fig. $4 D$, green curve), closely corresponding to the in vitro measurements. In vivo background current contains AMPA and NMDA receptormediated components that strongly differ in the resulting current correlation times $\tau_{\mathrm{NMDA}} \geq 50 \mathrm{~ms}$ and $\tau_{\mathrm{AMPA}} \leq 5 \mathrm{~ms}$ (Stern et al., 1992; Hestrin, 1993; Zito and Scheuss, 2007). For in vitro experiments, we therefore have used synthetic noise with a short $\left(\tau_{I}=\right.$ $5 \mathrm{~ms})$ or long $\left(\tau_{I}=50 \mathrm{~ms}\right)$ correlation time constants to match the in vivo noise constituents. To assess the effect of noise spectral composition on frequency response function, we normalized the vector strengths obtained in the three experiments to the response at the lowest frequency $r(f) / r(3 \mathrm{~Hz})$. The in vivo transfer function of neurons was enclosed by the transfer functions measured in vitro with $\tau_{I}=50 \mathrm{~ms}$ and $\tau_{I}=5 \mathrm{~ms}$ noise. This finding is consistent with the mixture of AMPA and NMDA receptor-mediated components present in the fluctuations of somatic net current in cortical neurons (Zito and Scheuss, 2007). These results demonstrate that cortical neurons in vitro and in vivo can encode input signals over a broad bandwidth, with cutoff frequency $f_{c}$ of $\sim 200-300 \mathrm{~Hz}$. For a linear system, this implies a response timescale of $\sim 1 /(2 \pi 200$ $\mathrm{Hz})<1 \mathrm{~ms}$, which is consistent with the fast timescale measured in our experiments with step stimuli (Figs. 1-3). Close correspondence between the response timescale estimated from frequency-domain experiments and the response speed directly measured in the time domain indicates that the assumption of linearity might be adequate for the description of mean evoked firing rate changes in our experiments.

\section{How many neurons are needed to rapidly detect a subtle step-like change of the mean input?}

To address this question, we assume a theoretical decoder that reports a step change of input current if the population firing rate falls outside the $95 \%$ confidence boundary of pre-step distribution (Fig. $5 A, B)$ (for details, see Materials and Methods). In our experiments, the background noise was uncorrelated across neurons; therefore, we are dealing with the idealized case of a population of $N$ uncorrelated neurons. Analysis of this idealized situation is both useful and necessary as a starting point for studying population responses of cortical neurons that exhibit weak crosscorrelations or decorrelated firing (Greenberg et al., 2008; Renart et al., 2010). Using the data from the step experiments (Fig. 1), we calculated for this decoder the probability of step detection as a function of the number of neurons that receive the common current step (equivalent to the number of realizations $N$ ) and time delay $T$ after the step onset. Figure 5, $C$ and $D$, illustrates that a small current step can be detected in the firing of 7400 neurons with $88 \%$ probability within the first millisecond after the step onset and with $>99 \%$ probability within $2 \mathrm{~ms}$ after the onset. The functional dependence of detection probability on the time elapsed after the step onset (Fig. $5 C$ ) or on the number of neurons $N$ in the population (Fig. $5 D$ ) can be well approximated by a tanh function, as can be expected from a decoder operating on two binomial distributions with different stationary rates. In the firing of 3700 neurons, the input current step is detected with a probability of $73 \%$ within $1 \mathrm{~ms}$ and with $>95 \%$ within $2 \mathrm{~ms}$ after the onset. Even in the firing of $\sim 2000$ neurons, the step is reliably detected $(>95 \%)$ within no more than $3 \mathrm{~ms}$. Similar results were obtained when the data from Figure 2 with low, intrinsic-only noise were used: a population of $\sim 2000$ neurons can reliably 
detect the step onset within no more than $3 \mathrm{~ms}$, and a population of 3700 neurons detects the step onset within $1 \mathrm{~ms}$ with $75 \%$ accuracy (data not shown). These results show that rapid detection of subtle input changes is robust over a broad range of amplitudes of membrane potential "noise" fluctuations. Thus, populations of 2000 or more cortical neurons can operate on an ultrafast timescale of 1-2 ms, conveying even minor input changes on a timescale significantly faster than the membrane time constant of the neurons.

\section{Discussion}

In this study, we demonstrate that populations of neurons in rat neocortex (1) can change their firing rate in response to small step-like changes of the mean input current very fast, within 1-2 $\mathrm{ms}$ and (2) can encode in their firing periodic signals up to frequencies of $200-300 \mathrm{~Hz}$, in vivo and in vitro. Results obtained in both time and frequency domains imply ultrafast, submillisecond timescale of population responses. We further show that (3) populations consisting of a few thousand neurons can reliably detect small changes of mean input within 1-2 ms.

\section{Ultrafast timescale of population response in theory and experiments}

Our results provide direct evidence that incoming signals, represented as changes of the mean input current, could be detected within $2 \mathrm{~ms}$ in the population firing of $\sim 2000$ neurons and within $1 \mathrm{~ms}$ in a population of $\sim 7000$ neurons. Although it is plausible to assume that low-pass filtering by the membrane could carry over to the firing rate dynamics, we show that neuronal ensembles are capable of operating on a timescale significantly shorter than the membrane time constant. The fast encoding is not limited to strong synapses (Galarreta and Hestrin 2001), but already a signal with an amplitude of typical cortical unitary postsynaptic current of $20 \mathrm{pA}$ (Stern et al., 1992; Hestrin, 1993) can change population firing rate on a millisecond timescale. Previously, such fast encoding has been considered possible only for the variance-coded signals (Silberberg et al., 2004) defining the prevailing dogma of the field that variance encoding is superior to mean encoding in transmitting fast signals. This dogma was primarily based on the observation that the firing rate response to a small mean current change develops slower than the response to a large change of the input variance and was partly supported by high cutoff frequency in responses to periodic variance modulation (Boucsein et al., 2009). Despite the fact that the timescale of the initial mean-induced firing rate transient has neither been quantified nor sufficiently resolved experimentally, the ultrafast transmission of rate encoded signals was deemed impossible. Here we refute this notion, showing that subtle changes of the mean input can be detected by populations of cortical neurons on a millisecond scale. Thus, mean-encoding channel and rate encoding in general can operate with subtle signals on an ultrafast timescale. We corroborate this conclusion by demonstrating that the population firing rate of neocortical neurons in vivo and in vitro can reliably encode frequencies up to $\sim 200-300 \mathrm{~Hz}$, which is $\sim 50$ times higher than the firing rate of individual neurons. These results resolve the contradiction between two recent studies that arrived at several-fold different estimates of cutoff frequency in responses of layer 5 pyramidal neurons to periodic mean modulated signals (Koendgen et al., 2008; Boucsein et al., 2009). Our results further extend these findings showing that (1) weak high-frequency modulation in the mean input can be reliably relayed by populations of layer $2 / 3$ pyramids, which mediate communication between cortical re- gions. (2) This input sensitivity is not restricted to in vitro conditions, but rather neocortical neurons in vivo under natural synaptic bombardment can reliably encode high-frequency inputs in their population firing rate. (3) The in vivo frequency response is close to that in vitro obtained for noise with correlation time constants of 5 and $50 \mathrm{~ms}$. Because in our experiments in the time domain (latency of responses to steps) and in the frequency domain (frequency response function) were performed under very similar conditions, i.e., on neurons of the same type, using injection of the current stimuli of similar amplitudes, with the same range of membrane potential fluctuations and the firing rates, we can directly compare the response timescale measured in these two paradigms. In a linear system, the response time of a simple low-pass filter is related to the cutoff frequency $f_{c}$ as $1 /\left(2 \pi f_{c}\right)$. The cutoff frequency $f_{c} \approx 200-300 \mathrm{~Hz}$ assessed in our experiments (Fig. 4) and in a recent study on layer 5 pyramidal neurons (Koendgen et al., 2008), corresponds to a response timescale $<1 \mathrm{~ms}$. This estimate is in agreement with the response timescale found in current step experiments (Figs. 1-3), indicating that the assumption of linear response where the cutoff frequency $f_{c}$ and response speed in the time domain are directly related might hold for our experimental conditions. Thus, coherent evidence from in vitro and in vivo measurements in the time and frequency domains supports the conclusion that cortical neuron populations can indeed communicate on an ultrafast millisecond timescale using mean-coded signals.

Previous theoretical work showed that a population of the most simple model neurons, LIF neurons (Tuckwell, 1988), can faithfully represent input signals of arbitrary frequency in changes of the firing rate (Brunel et al., 2001; Lindner and Schimansky-Geier, 2001; Naundorf et al., 2005a) and alter its firing rate instantaneously in response to a small step-like change of the mean or variance of the input current (Brunel et al., 2001). In contrast to the LIF model neurons, which are equipped with an instantaneous spike generation mechanism, the response timescale successively decreases in populations of conductance-based models if the timescales involved in spike generation are increased to capture the activation kinetics of sodium channels (Fourcaud-Trocmé et al., 2003). In all variations of conductance-based Hodgkin-Huxley-type models and their approximations studied so far, the response to fast varying inputs of frequencies $f$ higher than the firing rate of individual neurons is attenuated proportional to $1 / f$, thus limiting the reliably encoded frequency range (Fourcaud-Trocmé et al., 2003; Fourcaud-Trocmé and Brunel, 2005; Naundorf et al., 2005a). For an average firing rate of $1-5 \mathrm{~Hz}$, which is typical for cortical neurons, only input frequencies up to $\sim 10-20 \mathrm{~Hz}$ could be reliably encoded by conductancebased models (Fourcaud-Trocmé et al., 2003; Naundorf et al., 2005a). The attenuation of responses to signals beyond $f_{c}$ of $\sim 10-20$ Hz leads to a slow response timescale $(\sim 8-16 \mathrm{~ms})$ that is close to the membrane time constant (Fourcaud-Trocmé et al., 2003). Results reported here demonstrate that populations of cortical neurons in vitro and in vivo firing at $\sim 5 \mathrm{~Hz}$ can reliably encode low-amplitude input signals varying at frequencies up to $200-300 \mathrm{~Hz}$ and detect subtle changes of mean current on the corresponding ultrafast millisecond timescale. We propose to call this phenomenon the "Brunel effect" because such ultrafast responses were first predicted by Nicolas Brunel and coworkers and shown to be specific to integrate-andfire-type neuron models or more generally models with rapid spike dynamics (Brunel et al., 2001; Fourcaud-Trocmé et al., 2003; Naundorf et al., 2005a). We also confirmed the rapid population dynamics in response to changes of input variance predicted by such models. However, we observe these rapid firing rate changes only for large changes of input variance. We find that a threefold increase of the SD 
evoked a fast population rate transient, whereas a $50 \%$ increase of the SD did not lead to a measurable change of the population firing rate. According to shot noise theory, the input current variance $\left(\sigma^{2}\right)$ in a balanced cortical network is proportional to the average firing rate (van Kampen, 2007). This implies that a moderate 50\% increase of the SD necessitates a collective firing rate increase by $125 \%$, and a threefold increase of SD requires a dramatic ninefold increase of the average firing rate in a network, associated with corresponding increase of the metabolic energy demands.

\section{Changing the mean or the variance: two viable strategies for cortical communication?}

Comparison of the population firing rate responses to changes of the input mean or its variance revealed a substantial difference in the response dynamics. Even small changes of the mean input current induced an instantaneous change of population firing rate at the step onset and a robust change of the stationary firing rate. In contrast, only large changes in the input variance could affect the population firing rate, whereby the response was predominantly transient with little stationary component. This difference in response dynamics indicates the intriguing possibility of different computational strategies implemented in the neuronal communication channels that are using mean-coded and variance-coded signals. Population firing can be rapidly altered either by even a minor change of the input mean or by an extraordinarily large change of the input variance. Functionally, these two encoding schemes could serve different purposes: the ultrafast detection of small mean current changes could underlie fast cortical processing of rate-coded signals, whereas a variance encoding scheme could function as a filter that relays quickly only strong network perturbations.

\section{References}

Boucsein C, Tetzlaff T, Meier R, Aertsen A, Naundorf B (2009) Dynamical response properties of neocortical neuron ensembles: multiplicative versus additive noise. J Neurosci 29:1006-1010.

Brunel N, Chance FS, Fourcaud N, Abbott LF (2001) Effects of synaptic noise and filtering on the frequency response of spiking neurons. Phys Rev Lett 86:2186-2189.

Destexhe A, Rudolph M, Paré D (2003) The high-conductance state of neocortical neurons in vivo. Nat Rev Neurosci 4:739-751.

Ecker AS, Berens P, Keliris GA, Bethge M, Logothetis NK, Tolias AS (2010) Decorrelated neuronal firing in cortical micro circuits. Science 327:584-587.

Fourcaud-Trocmé N, Brunel N (2005) Dynamics of the instantaneous firing rate in response to changes in input statistics. J Comput Neurosci $18: 311-321$

Fourcaud-Trocmé N, Hansel D, van Vreeswijk C, Brunel N (2003) How spike generation mechanisms determine the neuronal response to fluctuating inputs. J Neurosci 23:11628-11640.

Galarreta M, Hestrin S (2001) Spike transmission and synchrony detection in networks of GABAergic interneurons. Science 292:2295-2299.

Gilbert CD, Wiesel TN (1979) Morphology and intracortical projections of functionally characterised neurones in the cat visual cortex. Nature 280:120-125.

Goldberg JM, Brown PB (1969) Response of binaural neurons of dog superior olivary complex to dichotic tonal stimuli: some physiological mechanisms of sound localization. J Neurophysiol 32:613-636.

Greenberg DS, Houweling AR, Kerr JN (2008) Population imaging of ongoing neuronal activity in the visual cortex of awake rats. Nat Neurosci 11:749-751.

Hestrin S (1993) Different glutamate receptor channels mediate fast excitatory synaptic currents in inhibitory and excitatory cortical neurons. Neuron 11:1083-1091.
Higgs MH, Spain WJ (2009) Conditional bursting enhances resonant firing in neocortical layer 2-3 pyramidal neurons. J Neurosci 29:1285-1299.

Jacobson GA, Diba K, Yaron-Jakoubovitch A, Oz Y, Koch C, Segev I, Yarom Y (2005) Subthreshold voltage noise of rat neocortical pyramidal neurons. J Physiol 564:145-160.

Joris PX, Schreiner CE, Rees A (2004) Neural processing of amplitudemodulated sounds. Physiol Rev 84:541-577.

Köndgen H, Geisler C, Fusi S, Wang XJ, Lüscher HR, Giugliano M (2008) The dynamical response properties of neocortical neurons to temporally modulated noisy inputs in vitro. Cereb Cortex 18:2086-2097.

Lindner B, Schimansky-Geier L (2001) Transmission of noise coded versus additive signals through a neuronal ensemble. Phys Rev Lett 86:2934-2937.

London M, Roth A, Beeren L, Häusser M, Latham PE (2010) Sensitivity to perturbations in vivo implies high noise and suggests rate coding in cortex. Nature 466:123-127.

Matsumura M, Chen D, Sawaguchi T, Kubota K, Fetz EE (1996) Synaptic interactions between primate precentral cortex neurons revealed by spike-triggered averaging of intracellular membrane potentials in vivo. J Neurosci 16:7757-7767.

Naundorf B, Geisel T, Wolf F (2005a) Action potential onset dynamics and the response speed of neuronal populations. J Comput Neurosci 18:297-309.

Naundorf B, Geisel T, Wolf F (2005b) Dynamical response properties of a canonical model for type-I membranes. Neurocomputing 65:421-428.

Okun M, Lampl I (2008) Instantaneous correlation of excitation and inhibition during ongoing and sensory-evoked activities. Nat Neurosci 11:535-537.

Ostojic S, Brunel N, Hakim V (2009) How connectivity, background activity, and synaptic properties shape the cross-correlation between spike trains. J Neurosci 29:10234-10253.

Rauch A, La Camera G, Luscher HR, Senn W, Fusi S (2003) Neocortical pyramidal cells respond as integrate-and-fire neurons to in vivo-like input currents. J Neurophysiol 90:1598-1612.

Renart A, de la Rocha J, Bartho P, Hollender L, Parga N, Reyes A, Harris KD (2010) The asynchronous state in cortical circuits. Science 327:587-590.

Silberberg G, Bethge M, Markram H, Pawelzik K, Tsodyks M (2004) Dynamics of population rate codes in ensembles of neocortical neurons. J Neurophysiol 91:704-709.

Stanford TR, Shankar S, Massoglia DP, Costello MG, Salinas E (2010) Perceptual decision making in less than 30 milliseconds. Nat Neurosci 13:379-385.

Steinmetz PN, Manwani A, Koch C, London M, Segev I (2000) Subthreshold voltage noise due to channel fluctuations in active neuronal membranes. J Comput Neurosci 9:133-148.

Stern P, Edwards FA, Sakmann B (1992) Fast and slow components of unitary EPSCs on stellate cells elicited by focal stimulation in slices of rat visual cortex. J Physiol 449:247-278.

Swadlow HA, Hicks TP (1996) Somatosensory cortical efferent neurons of the awake rabbit: latencies to activation via supra- and subthreshold receptive fields. J Neurophysiol 75:1753-1759.

Thorpe S, Fize D, Marlot C (1996) Speed of processing in the human visual system. Nature 381:520-522.

Tuckwell H (1988) Introduction to theoretical neurobiology. Cambridge, UK: Cambridge UP.

van Kampen NG (2007) Stochastic processes in physics and chemistry, Ed 3. Amsterdam: Elsevier.

Volgushev M, Vidyasagar TR, Chistiakova M, Yousef T, Eysel UT (2000) Membrane properties and spike generation in rat visual cortical cells during reversible cooling. J Physiol 522:59-76.

Volgushev M, Pernberg J, Eysel UT (2002) A novel mechanism of response selectivity of cortical neurons. J Physiol 540:307-320.

Volgushev M, Chauvette S, Mukovski M, Timofeev I (2006) Precise longrange synchronization of activity and silence in neocortical neurons during slow-wave sleep. J Neurosci 26:5665-5672.

Zheng Y, Escabí MA (2008) Distinct roles for onset and sustained activity in the neuronal code for temporal periodicity and acoustic envelope shape. Neuroscience 28:14230-14244.

Zito K, Scheuss V (2007) NMDA receptor function and physiological modulation. In: The new encyclopedia of neuroscience (Squire L, ed), pp 276-283. Oxford, UK: Academic. 